\title{
Confinement Effects in Low-Dimensional Lead lodide Perovskite Hybrids
}

\author{
Machteld E. Kamminga, ${ }^{\dagger}$ Hong-Hua Fang, ${ }^{\dagger}$ Marina R. Filip, ${ }^{\ddagger}$ Feliciano Giustino, ${ }^{\ddagger}$ Jacob Baas, ${ }^{\dagger}$ \\ Graeme R. Blake, ${ }^{\dagger}$ Maria Antonietta Loi, ${ }^{\dagger}$ and Thomas T. M. Palstra*, ${ }^{\dagger}$ \\ ${ }^{\dagger}$ Zernike Institute for Advanced Materials, University of Groningen, Nijenborgh 4, 9747 AG Groningen, The Netherlands \\ ${ }^{\ddagger}$ Department of Materials, University of Oxford, Parks Road, Oxford OX1 3PH, United Kingdom
}

\section{Supporting Information}

ABSTRACT: We use a layered solution crystal growth technique to synthesize high-quality single crystals of phenylalkylammonium lead iodide organic/inorganic hybrid compounds. Single-crystal X-ray diffraction reveals low-dimensional structures consisting of inorganic sheets separated by bilayers of the organic cations. The shortest alkyls yield two-dimensional structures consisting of inorganic sheets of corner-sharing $\mathrm{PbI}_{6}$-octahedra. However, the longer alkyls induce both corner- and face-sharing of the $\mathrm{PbI}_{6}$ octahedra, and form new compounds. Density functional theory calculations including spin-orbit coupling show quantum confinement in two dimensions

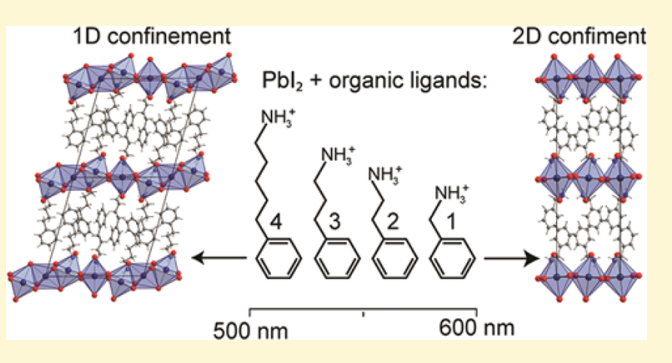
for the shorter alkyls, and in one dimension for the longer alkyls, respectively. The face-sharing $\mathrm{PbI}_{6}$-octahedra create a confinement leading to effectively one-dimensional behavior. These confinement effects are responsible for the observed peak shifts in photoluminescence for the different phenylalkylammonium lead iodide hybrids. Our results show how the connectivity of the octahedra leads to confinement effects that directly tune the optical band gap.

\section{INTRODUCTION}

Initially used as a sensitizer in dye-solar cells, ${ }^{1} \mathrm{CH}_{3} \mathrm{NH}_{3} \mathrm{PbI}_{3}$ has attracted much attention as an efficient absorber material in planar heterojunction solar cell devices. ${ }^{2-7}$ Very high powerconversion efficiencies of up to $22.1 \%^{8}$ have been reported for lead iodide-based materials. $\mathrm{CH}_{3} \mathrm{NH}_{3} \mathrm{PbI}_{3}$ belongs to the group of organic/inorganic hybrid materials that consist of organic cations and halogenometalates. In addition to solar light conversion, other optoelectronic applications of this class of materials have recently been reported owing to their outstanding optical properties. Light emitting diodes, ${ }^{9,10}$ lasers $^{11,12}$ and photodetectors ${ }^{13}$ have extended not only the scope of these materials but also the spectrum of interesting band gaps. Substitution of either the organic or inorganic component will greatly influence the polarizability and optical band gap, crucial for photovoltaics. As the valence band is formed by hybridized $\mathrm{Pb}-6 s$ and halide- $p$ orbitals and the conduction band is primarily $\mathrm{Pb}-6 p$ in character, ${ }^{14}$ substitution of the (metal) halide affects the band gap. ${ }^{15}$ However, substitution of the organic component changes the band gap as well, because the size of the organic cation alters the inorganic framework responsible for the size of the band gap. ${ }^{16-18}$ Thus, the band gap of organic/inorganic hybrids is related to the structure of the inorganic framework that can be controlled by the organic part. Understanding how the band gap can be altered allows the band gap of hybrid perovskite materials to be tuned for desired applications.

In addition to three-dimensional (3D) organic/inorganic hybrids, two-dimensional (2D) hybrids are of particular interest. Recently, Smith et al. ${ }^{19}$ reported a first-generation solar cell device consisting of a layered hybrid perovskite absorber. This device benefited from possible fabrication under ambient conditions and exhibited enhanced moisture stability compared to the $3 \mathrm{D} \mathrm{CH}_{3} \mathrm{NH}_{3} \mathrm{PbI}_{3}$ analogue. In most cases, these $2 \mathrm{D}$ hybrid structures consist of single $\langle 100\rangle$-terminated perovskite sheets separated by bilayers of alkylammonium cations and held together through van der Waals interactions between the alkyl chains. ${ }^{20}$ These compounds form a quantum well structure, in which 2D semiconductor layers are separated by organic spacers. As a result of this quantum confinement effect and enhanced dielectric confinement, stable excitons with large binding energies can be formed. ${ }^{21}$ Whereas the high exciton binding energy is disadvantageous for solar cell device operation, these $2 \mathrm{D}$ structures exhibit conduction within the layers $^{20}$ and have potential applications in light emission. 9,10 Although the small organic cations are free to rotate in the $3 \mathrm{D}$ perovskite lattice, they are particularly rigid in the $2 \mathrm{D}$ structure due to van der Waals interactions and possible $\pi-\pi$ interactions in the case of phenylalkyl chains. As a result, a $2 \mathrm{D}$ layer of positively charged amine groups is formed. In combination with the neighboring layer of negatively charged iodide anions, a polar surface is formed between the organic and inorganic layers. Even though 2D hybrid structures have fewer conduction pathways compared to $3 \mathrm{D}$ structures, they benefit from their enhanced polarizability. In fact, ferroelectricity was found in structurally similar chlorocuprates. ${ }^{22}$ Moreover, 2D

Received: February 26, 2016

Revised: June 13, 2016 
materials can find applications in field effect transistors, light emitting diodes, lasers and detectors. Thus, 2D hybrid structures allow for tunability of their properties, as the organic cations are not restricted to a small size.

Here we investigate how the band gap of low-dimensional hybrids can be tuned by substituting phenylalkylamines for methylammonium in $\mathrm{CH}_{3} \mathrm{NH}_{3} \mathrm{PbI}_{3}$. Methylammonium is a rather small cation that fits in the 12 -fold iodine-coordinated interstitial holes created by the extended $3 \mathrm{D}$ network formed by corner-sharing $\mathrm{PbI}_{6}$-octahedra. As only small organic cations consisting of three or fewer $\mathrm{C}-\mathrm{C}$ or $\mathrm{C}-\mathrm{N}$ bonds are expected to fit in this $3 \mathrm{D}$ structure, ${ }^{23}$ we introduce larger phenylalkylamines to obtain $2 \mathrm{D}$ hybrid structures. Moreover, we choose organic ligands with phenyl groups, as interactions between the aromatic rings provide a positive contribution to the formation of the hybrid structures through self-assembly. We use four different phenylalkylammonium cations with alkyl chains that are one to four carbons long. The single crystals obtained in our study are stable under ambient and low-humidity conditions, and no degradation was observed over the course of months. Single-crystal XRD results reveal that the two compounds with the shortest phenylalkylammonium cations have structures that consist of inorganic layers of corner-sharing $\mathrm{PbI}_{6}$-octahedra separated by bilayers of the organic cations. ${ }^{24-27}$ This 2D confinement effect is well-known to influence the band gap. ${ }^{28,29}$ However, the two compounds with the longest phenylalkylammonium cations form new structures wherein the inorganic layers are formed by both corner- and face-sharing $\mathrm{PbI}_{6}$ octahedra. Our study of the electronic structures using density functional theory including relativistic spin-orbit coupling effects (hereafter referred to as DFT+SOC) show that increasing the length of the organic ligands results in band structure features consistent with confinement effects from 3D to $2 \mathrm{D}$ and effectively $1 \mathrm{D}$ as caused by the formation of facesharing $\mathrm{PbI}_{6}$-octahedra, in agreement with shifts in our photoluminescence data. Thus, although it is a general guideline that $2 \mathrm{D}$ confinement and the size of the organic cation and halide have a templating influence on the band gap, we show that local structural features can cause the surprising effect of $1 \mathrm{D}$ confinement. Our results show how confinement effects enable direct tuning of the optical band gap in organic/ inorganic hybrid materials and indicate the design rules for obtaining a large library of hybrid perovskites with interesting optoelectronic properties.

\section{EXPERIMENTAL SECTION}

Crystal Growth. Single crystals of organic/inorganic hybrid perovskites were grown at room temperature using a layered solution technique as previously reported by Mitzi. ${ }^{30}$ The amines and halogenometalates were dissolved in separate solutions. As the different solutions have large differences in density, a sharp interface is formed when the two components are combined. Single crystals were formed due to slow diffusion at the interface.

Procedure. $74 \mathrm{mg}(0.16 \mathrm{mmol})$ of $\mathrm{PbI}_{2}$ (Sigma-Aldrich; 99\%) was dissolved in $3.0 \mathrm{~mL}$ of concentrated ( $57 \mathrm{wt} \%$ ) aqueous hydriodic acid (Sigma-Aldrich; 99.95\%). This light-yellow mixture was poured into a glass test tube (size $18 \times 150 \mathrm{~mm})$. A syringe with needle was used to make sure that the mixture was placed at the bottom of the tube. 3.0 $\mathrm{mL}$ of absolute methanol (Lab-Scan; anhydrous, $99.8 \%$ ) was carefully place on top of the $\mathrm{PbI}_{2} / \mathrm{HI}$ mixture using another syringe and needle, without mixing the solutions. A sharp interface was formed between the two layers due to the large difference in densities $(0.791$ and 1.701 $\mathrm{g} / \mathrm{mL}$ for methanol and concentrated aqueous hydriodic acid, respectively). Four types of phenylalkylamine ligands were used: benzylamine (Sigma-Aldrich; 99\%), 2-phenethylamine (Sigma-Aldrich;
99\%), 3-phenyl-1-propylamine (Sigma-Aldrich; 98\%) and 4-phenyl-1butylamine (Sigma-Aldrich). The phenylalkylammonium solutions were added in great excess by gently adding 15 droplets, using a glass pipette, on top of the methanol layer. The test tubes were covered with aluminum foil and kept in a fume hood under ambient conditions. After $24 \mathrm{~h}$, a small number of $(\mathrm{PEA})_{2} \mathrm{PbI}_{4}$ and $(\mathrm{PBA})_{3} \mathrm{~Pb}_{2} \mathrm{I}_{7}$ crystals were formed at the interface and gathered at the bottom of the tube. The (PMA) $)_{2} \mathrm{PbI}_{4}$ crystals started to form on the second day and the (PPA) ${ }_{3} \mathrm{~Pb}_{2} \mathrm{I}_{7}$ crystals followed half a day later. The crystals were collected after 5 days as the interface between the two layers rapidly degrades over the course of 1 week. The crystals were washed three times with diethyl ether (Avantor) after carefully pouring the contents of the test tubes through a filter. Besides being a colorless solvent with a very low vapor pressure, the crystals do not dissolve in diethyl ether, which makes it an excellent solvent for washing the crystals. After drying under ambient conditions, all crystals were stored in a drybox. The obtained high-quality crystals are bright orange (for ( $\mathrm{PMA})_{2} \mathrm{PbI}_{4}$ and slightly lighter for (PEA) ${ }_{2} \mathrm{PbI}_{4}$ ) and bright yellow (for $(\mathrm{PPA})_{3} \mathrm{~Pb}_{2} \mathrm{I}_{7}$ and $\left.(\mathrm{PBA})_{3} \mathrm{~Pb}_{2} \mathrm{I}_{7}\right)$ in color. The (PMA $)_{2} \mathrm{PbI}_{4}$ and (PEA) ${ }_{2} \mathrm{PbI}_{4}$ crystals were platelets of roughly $0.6 \mathrm{~mm}$ in diameter and the $(\mathrm{PPA})_{3} \mathrm{~Pb}_{2} \mathrm{I}_{7}$ and $(\mathrm{PBA})_{3} \mathrm{~Pb}_{2} \mathrm{I}_{7}$ crystals were very thin needles of around $1.3 \mathrm{~mm}$ long.

X-ray Diffraction. Powder X-ray diffraction (XRD) data were collected using a Bruker D8 Advance diffractometer in BraggBrentano geometry and operating with $\mathrm{Cu} \mathrm{K} \alpha$ radiation. The data were fitted using the EXPO ${ }^{31}$ and GSAS $^{32}$ software suites. Single crystal XRD measurements were performed using a Bruker D8 Venture diffractometer equipped with a Triumph monochromator and a Photon 100 area detector, operating with $\mathrm{Mo} \mathrm{K} \alpha$ radiation. A $0.3 \mathrm{~mm}$ nylon loop and cryo-oil were used to mount the crystals. The crystals were cooled with a nitrogen flow from an Oxford Cryosystems Cryostream Plus. Data processing was done using the Bruker Apex II software and the SHELX97 software $^{33}$ was used for structure solution and refinement.

Photoluminescence Measurements. The photoluminescence (PL) measurements were performed by exciting the single crystals at approximately $400 \mathrm{~nm}$ by the second harmonic of a mode-locked TiSapphire laser (Mira 900, Coherent). The PL spectra were recorded by a Hamamatsu CCD camera. A calibrated light source was used to correct the measurements with respect to the spectral response of the setup. The time-resolved photoluminescence spectra were measured with the Hamamatsu CCD camera in synchroscan mode. The same mode-locked femtosecond laser with a repetition rate of $76 \mathrm{MHz}$ was used as the excitation source.

Computational Methods. All calculations were performed within density functional theory (DFT) ${ }^{34}$ in the local density approximation $(\mathrm{LDA})^{35}$ including relativistic spin-orbit coupling effects. All calculations were performed using the experimental lattice parameters and atomic positions. For ( $\mathrm{PMA})_{2} \mathrm{PbI}_{4}$, the unit cells consist of 168 atoms and 512 electrons, whereas for $(\mathrm{PPA})_{3} \mathrm{~Pb}_{2} \mathrm{I}_{7}, 648$ atoms and 1936 electrons are included. All calculations were performed using the Quantum Espresso simulation package. ${ }^{36}$ For all atomic components, the ultrasoft pseudopotentials ${ }^{37}$ available in the Quantum Espresso library were used. In the case of $\mathrm{Pb}$ and $\mathrm{I}$, fully relativistic pseudopotentials were used; whereas for $\mathrm{C}, \mathrm{N}$ and $\mathrm{H}$, scalar- and nonrelativistic pseudopotentials were used. For the calculation of the charge density, a plane wave cutoff of $40 \mathrm{Ry}$ and a charge density cutoff of $200 \mathrm{Ry}$ were used. The Brillouin zone was sampled using a $2 \times 2 \times$ 2 Brillouin-zone grid centered at $\Gamma$ for both (PMA) ${ }_{2} \mathrm{PbI}_{4}$ and (PPA) ${ }_{3} \mathrm{~Pb}_{2} \mathrm{I}_{7}$. Structural models were rendered using VESTA. ${ }^{38}$

\section{RESULTS AND DISCUSSION}

We investigate the structure and photoluminescence response of lead iodide perovskite-like organic/inorganic hybrids with aromatic ligands of increasing length. We use four types of phenylalkylammonium cations, where the phenyl (P) and ammonium (A) groups are separated by a methyl (M), ethyl (E), propyl (P) or butyl (B) group, i.e., $\mathrm{C}_{6} \mathrm{H}_{5}\left(\mathrm{CH}_{2}\right)_{n} \mathrm{NH}_{3}{ }^{+}$with $n=1-4$. We abbreviate these organic ligands as PMA, PEA, 
a) $(\mathrm{PMA})_{2} \mathrm{Pbl}_{4}$

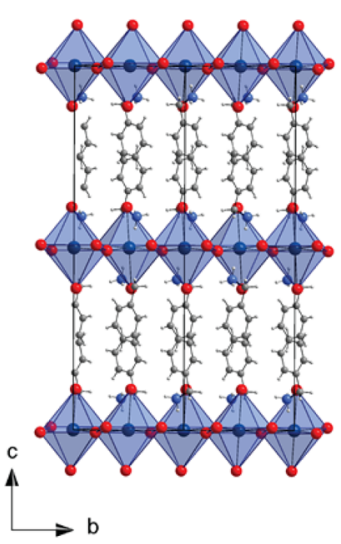

b)

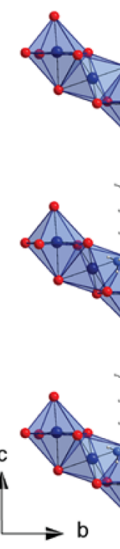

$(\mathrm{PPA})_{3} \mathrm{~Pb}_{2} \mathrm{I}_{7}$

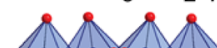

c)

(PBA) $)_{3} \mathrm{~Pb}_{2} \mathrm{I}_{7}$

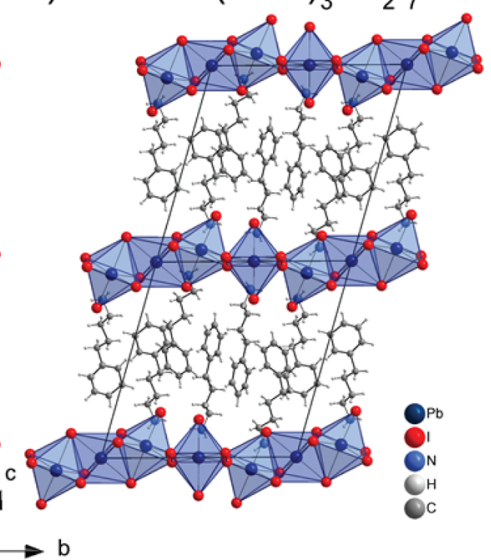

Figure 1. Polyhedral model of three structural motifs at $100 \mathrm{~K}$, projected along the $[100]$ direction. (a) Crystal structure of $\left(\mathrm{PMA}_{2} \mathrm{PbI}_{4}\right.$. The structure of $(\mathrm{PEA})_{2} \mathrm{PbI}_{4}$ is similar. (b) Crystal structure of $(\mathrm{PPA})_{3} \mathrm{~Pb}_{2} \mathrm{I}_{7}$. (c) Crystal structure of $(\mathrm{PBA})_{3} \mathrm{~Pb}_{2} \mathrm{I}_{7}$.

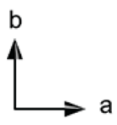

a)

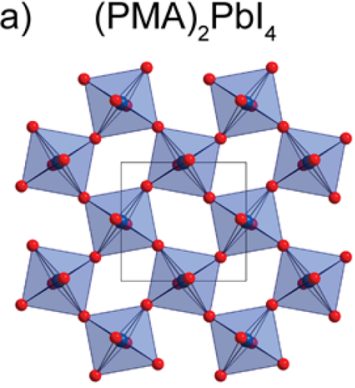

b) (PEA) ${ }_{2} \mathrm{Pbl}_{4}$

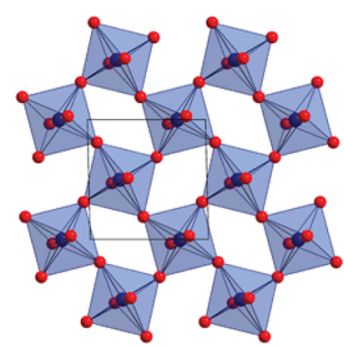

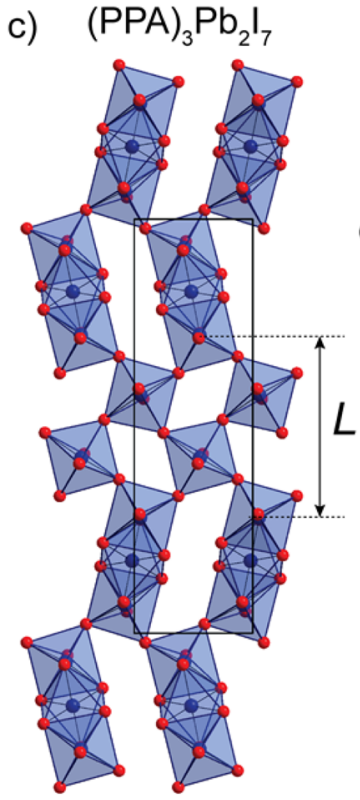

d)

$(\mathrm{PBA})_{3} \mathrm{~Pb}_{2} \mathrm{I}_{7}$

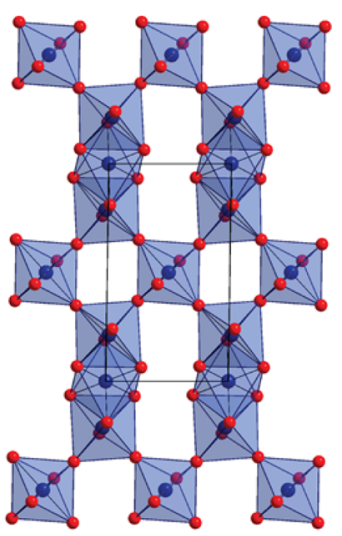

Figure 2. Polyhedral model of single inorganic sheets projected along the [001] direction. (a) $(\mathrm{PMA})_{2} \mathrm{PbI}_{4},(\mathrm{~b})(\mathrm{PEA})_{2} \mathrm{PbI}_{4},(\mathrm{c})\left(\mathrm{PPA}_{3} \mathrm{~Pb}_{2} \mathrm{I}_{7},(\mathrm{~d})\right.$ $(\mathrm{PBA})_{3} \mathrm{~Pb}_{2} \mathrm{I}_{7} . L$ is the length of the region created by corner-sharing $\mathrm{PbI}_{6}$-octahedra along the $[010]$ direction in $(\mathrm{PPA})_{3} \mathrm{~Pb} \mathrm{I}_{2}$. The organic molecules are not shown for clarity.

PPA and PBA, respectively. These organic groups are relatively large and form structures with inorganic perovskite-like layers. In our case, $\mathrm{PbI}_{4}{ }^{2-}$ layers of corner-sharing and/or face-sharing octahedra separated by bilayers of the organic cations are formed. Previous work reports the synthesis and optical studies of PMA-, ${ }^{24,34,35}$ PEA- $^{24-27,35,36}$ and PPA-based ${ }^{26}$ lead iodide hybrids. However, Zhang et al. ${ }^{26}$ did not show structural data of the PPA-based lead iodide compound they made and assumed it to be isostructural with the PMA- and PEA-based lead iodide structures. Our single-crystal XRD studies confirm that the PMA- and PEA-based crystals adopt the reported (PMA) ${ }_{2} \mathrm{PbI}_{4}$ and $(\mathrm{PEA})_{2} \mathrm{PbI}_{4}$ structures, as shown in Figure 1a. However, the PPA-based crystals adopt a different structure, as shown in Figure $1 \mathrm{~b}$. Billing and Lemmerer ${ }^{37}$ investigated the crystal structure of PPA lead iodide and found the structural formula to be $(\mathrm{PPA})_{4} \mathrm{~Pb}_{3} \mathrm{I}_{10}$. It consists of face-sharing trimers of $\mathrm{PbI}_{6}$ octahedra that are corner-shared to form the inorganic layer. Similar stoichiometries have been observed in
$\left(\mathrm{PhMe}_{3} \mathrm{~N}\right)_{4} \mathrm{~Pb}_{3} \mathrm{Br}_{10}{ }^{38}$ and $\left(\mathrm{PhMe}_{3} \mathrm{~N}\right)_{4} \mathrm{Sn}_{3} \mathrm{I}_{10},{ }^{39}$ but with an alternative packing of the trimeric building blocks. Notably, our single crystals adopt a completely different and new crystal structure with the composition ( $\mathrm{PPA})_{3} \mathrm{~Pb}_{2} \mathrm{I}_{7}$. The structure consists of inorganic sheets that are separated by bilayers of the organic PPA. The inorganic sheets have a remarkable structure as they combine corner-sharing with face-sharing $\mathrm{PbI}_{6}$ octahedra. The face-sharing induces the formation of trimers, where a single $\mathrm{PbI}_{6}$-octahedron shares both faces with a neighboring octahedron. Figure $1 \mathrm{~b}$ shows that the layers are not flat due to the stiffness of the face-sharing $\mathrm{Pb}_{3} \mathrm{I}_{10}$-trimers. The face-sharing $\mathrm{Pb}_{3} \mathrm{I}_{10}$-trimers are linked to single $\mathrm{PbI}_{6}$-octahedra by corner sharing. Although the compounds mentioned above $^{37-39}$ consist of $\left[\mathrm{M}_{3} \mathrm{X}_{10}\right]^{4-}$ units that are corner-shared ( $\mathrm{M}$ is a divalent metal and $\mathrm{X}$ is a halide), the compound we present here is constructed from both $\left[\mathrm{M}_{3} \mathrm{X}_{10}\right]^{4-}$ and $\left[\mathrm{MX}_{4}\right]^{2-}$ units that are corner-shared. Thus, we obtain a different crystal structure than Billing and Lemmerer, ${ }^{37}$ using different 
precursors and no heating while synthesizing. It represents a new structure type. The structural data and refinement parameters are listed in Table $\mathrm{S} 1$ in the Supporting Information. The unit cell of (PPA) ${ }_{3} \mathrm{~Pb}_{2} \mathrm{I}_{7}$ is orthorhombic and the structure adopts the noncentrosymmetric polar $P_{c a 2}$ space group with an inversion twin (population is around 13\%). This space group is maintained over our measured temperature range from 100 to $400 \mathrm{~K}$. Table S2 shows how the lattice parameters vary and how the volume increases with temperature. Our DSC measurement reveals that the compound degrades at temperatures above $400 \mathrm{~K}$, by showing an irreversible process. Figure 1 shows the three different structural motifs observed in our crystals. Here, we report the photoluminescence and band structure calculations. Other physical properties will be reported separately.

We observe that the PBA-based lead iodide hybrid is quite similar and forms the (PBA) ${ }_{3} \mathrm{~Pb}_{2} \mathrm{I}_{7}$ structure. Figure $1 \mathrm{c}$ shows the projection along the [100] direction, whereas Figure $2 \mathrm{~d}$ shows a single inorganic sheet of this structure. It contains the same combination of corner-sharing and face-sharing $\mathrm{PbI}_{6}$ octahedra (Figure 2c), but in a different pattern due to different crystal symmetry. This structure adopts the triclinic $P \overline{1}$ space group and has inversion centers at the middle, corners and edges of the unit cell. This is also a new structure, and it belongs to the same structure class as the PPA-based hybrids with formula $\mathrm{A}_{3} \mathrm{M}_{2} \mathrm{X}_{7}$. Further structural data and refinement parameters are listed in Table $\mathrm{S} 1$ in the Supporting Information. The crystal structure solutions are confirmed by fitting single-crystal XRD data to powder XRD spectra of $(\mathrm{PPA})_{3} \mathrm{~Pb}_{2} \mathrm{I}_{7}$ and $(\mathrm{PBA})_{3} \mathrm{~Pb}_{2} \mathrm{I}_{7}$, as shown in Figure S2. Figure S3 shows the presence of high-temperature phase transitions, indicated by reversible DSC peaks. However, as one of the organic cations is already particularly disordered at $100 \mathrm{~K}$ and the $R$-factors are relatively high, as discussed in the caption of Table S1, no structural analysis of the higher temperature phases is presented here.

The crystal structure of $(\mathrm{PEA})_{2} \mathrm{PbI}_{4}$ is reported to be monoclinic with space group $\mathrm{C} 2 / \mathrm{m},{ }^{27}$ but with iodide ions that are split over two positions. As our work focuses on the formation of the inorganic lattice, we chose to refine the structure in the lower symmetry triclinic space group $P 1$. This allows the positions of the ions to be determined without restricting their positions by symmetry. Therefore, our structure determination of the inorganic sheets is accurate, but we do not present the full structure because the positions and orientations of the organic molecules could not be determined with sufficient certainty.

For the four compounds presented in this work, the main structurally driving interaction is the hydrogen bonding between the iodides and the amine groups. Although this interaction effectively forms the structure, the tails of the organic cations are left to arrange as possible. For all compounds, we do not observe any $\pi-\pi$ stacking, the phenyl groups are arranged almost perpendicular to each other. In $(\mathrm{PBA})_{3} \mathrm{~Pb}_{2} \mathrm{I}_{7}$, however, the tails of organic cations are particularly disordered and no clear arrangement is observed.

In Figure 3, we show the photoluminescence spectra of single crystals of all four layered lead iodide hybrids. Our results clearly indicate a shift of the peak maxima toward the blue when the length of the ligand is increased. Thus, systematically increasing the length from PMA to PBA induces a significant blue-shift. This clearly corresponds to the observation that the $(\mathrm{PMA})_{2} \mathrm{PbI}_{4}$ crystals are orange, the $(\mathrm{PEA})_{2} \mathrm{PbI}_{4}$ crystals are

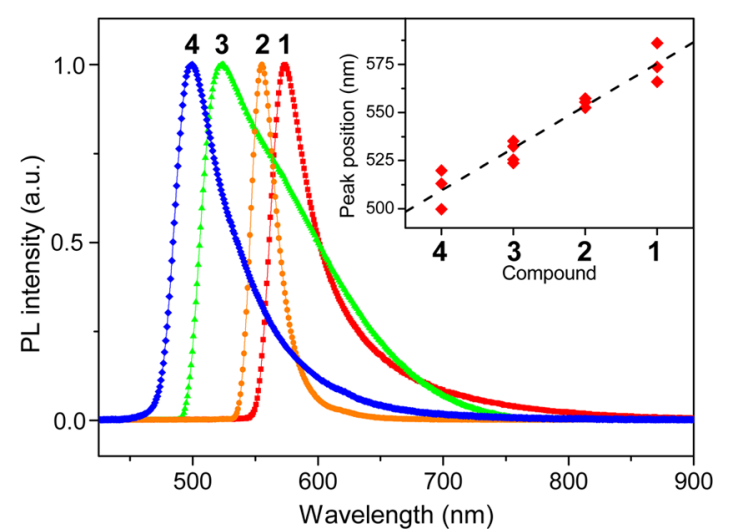

Figure 3. Selected normalized photoluminescence (PL) spectra for layered lead iodide hybrids with ligands of increasing length (1-4). Compounds 1-4 refer to (PMA) $)_{2} \mathrm{PbI}_{4},(\mathrm{PEA})_{2} \mathrm{PbI}_{4},(\mathrm{PPA})_{3} \mathrm{~Pb}_{2} \mathrm{I}_{7}$ and $(\mathrm{PBA})_{3} \mathrm{~Pb}_{2} \mathrm{I}_{7}$, respectively. The inset shows the photoluminescence peak positions for multiple crystals. The dotted trend line shows the blue-shift with increasing alkyl chain length.

yellow-orange, and the (PPA $)_{3} \mathrm{~Pb}_{2} \mathrm{I}_{7}$ and $(\mathrm{PBA})_{3} \mathrm{~Pb}_{2} \mathrm{I}_{7}$ crystals are yellow. The photoluminescence spectra are asymmetric toward lower energies. We observe that the presence of more complex inorganic sheets containing face-sharing $\mathrm{PbI}_{6}$-octahedra leads to an enhanced broadening of the photoluminescence. We ascribe this to the possible presence of defects and vacancies, which drive recombination in these more complex crystals. This can result in interstitial bands, giving rise to transitions at lower energy. Moreover, this is confirmed by the time-resolved decay dynamics which are fitted with biexponential and triexponential functions (Figure S4 in the Supporting Information). Such multiexponential decay implies the presence of defect states or shallow trapping levels in the grain boundaries of the crystals, which frequently behave as nonradiative recombination centers. We determine the photoluminescence peak positions by assuming a Gaussian line shape. The inset of Figure 3 shows that there is a distribution of peak positions for different crystals of each type. Such variation can be caused by differences in thickness, quality and concentration of intrinsic defects even within a single batch of crystals. Thus, multiple data points are plotted in the inset. The dotted trend line clearly shows that, even when taking natural variation into account, there is a clear blue-shift with increasing the organic ligand length from PMA to PBA.

Table 1 shows the conversion of the PL peaks into band gaps for the compounds in this work, placed into perspective with selected band gaps of related $3 \mathrm{D}$ and $2 \mathrm{D}$ organic/inorganic perovskite hybrid compounds. Although the inset in Figure 3 shows that there is a modest variation between different single crystals, the reported values also show a variation in band gap values for each type of single crystal. For $\mathrm{MASnI}_{3}$, it was even found to depend on the synthesis method used. ${ }^{40}$ A distinction is made between single crystals and thin films as their defects and trap densities can be different, resulting in observations of different band gaps in both morphologies. ${ }^{41}$ The focus of our work is on single crystals and a full study of thin film formation is beyond the scope of our work. Our single-crystal XRD results show that the compounds containing the two shortest organic ligands grow in sheets, perpendicular to the $c$-axis. Other studies have shown that films form in the same manner, with the $c$-axis perpendicular to the film. ${ }^{29,36}$ However, the new compounds with the longest organic ligands grow as needles, 
Table 1. Band Gaps (in eV) of Selected 3D and 2D Organic/ Inorganic Perovskite Hybrids, Measured for Single Crystals and Thin Films ${ }^{a}$

\begin{tabular}{lll} 
& \multicolumn{1}{c}{ single crystal } & \multicolumn{1}{c}{ thin film } \\
$\mathrm{MAPbI}_{3}$ & $1.51,^{42} 1.52,^{40} 1.61^{41,43}$ & $1.57^{16,44}$ \\
$\mathrm{MAPbBr}_{3}$ & $2.18^{42}$ & $2.33,^{45} 2.35^{46}$ \\
$\mathrm{MAPbCl}_{3}$ & & $3.04^{45}$ \\
$(\mathrm{MA})_{2} \mathrm{PbI}_{2}(\mathrm{SCN})_{2}$ & $2.14^{47}$ & $2.11,^{48} 2.33^{47}$ \\
$\mathrm{MASnI}_{3}$ & $1.21-1.35^{40}$ & $1.63^{45}$ \\
$\mathrm{MASnBr}_{3}$ & & $2.15^{45}$ \\
$\mathrm{FAPbI}_{3}$ & $1.45^{40}$ & $1.48^{16}$ \\
$\mathrm{CsPbI}$ & & $1.73^{16}$ \\
$(\mathrm{PMA})_{2} \mathrm{PbI}_{4}$ & $2.12-2.19$ & \\
$(\mathrm{PEA})_{2} \mathrm{PbI}_{4}$ & $2.22-2.24$ & $2.37,^{35} 2.38,,^{36} 2.39^{45}$ \\
$(\mathrm{PEA})_{2} \mathrm{PbBr}_{4}$ & & $3.05^{45}$ \\
$(\mathrm{PEA})_{2} \mathrm{PbCl}_{4}$ & & $3.63^{45}$ \\
$(\mathrm{PEA})_{2} \mathrm{SnI}_{4}$ & & $1.99^{45}$ \\
$(\mathrm{PPA})_{3} \mathrm{~Pb}_{2} \mathrm{I}_{7}$ & $2.32-2.37$ & \\
$(\mathrm{PBA})_{3} \mathrm{~Pb}_{2} \mathrm{I}_{7}$ & $2.39-2.48$ & \\
\hline
\end{tabular}

${ }^{a}$ Band gaps are determined from the $\mathrm{PL}$ maxima given in the references. MA and FA are used to abbreviate methylammonium and formamidinium, respectively.

with the longest axis being the $a$-axis. As a result, we cannot speculate about the film formation as preferential orientations are expected to play a role. Here we present design rules for tuning the band gap by changing the organic ligand. Table 1 shows various trends in how the band gap alters when the compound is changed. The effect of a smaller halide increases the band gap by varying metal-halide-metal angles, as discussed below, and the introduction of longer phenylalkyl ligands increases the band gap by the $2 \mathrm{D}$ confinement effect. Although these design rules are commonly understood, our work shows an additional design rule coming from the formation of face-sharing $\mathrm{PbI}_{6}$-octahedra as discussed below.

The large separation of the inorganic sheets by the organic cations creates a confinement effect from $3 \mathrm{D}$ to $2 \mathrm{D}$ and makes it valid to consider the electronic response from only a single $2 \mathrm{D}$ sheet. ${ }^{49}$ Because the organic cations do not significantly participate in the frontier orbitals, the system can be further simplified to the inorganic lattice, as in Figure 2. The length of the alkyl chain strongly influences the bonding in the inorganic part. Figure $2 \mathrm{a}, \mathrm{b}$ shows that the in-plane $\mathrm{Pb}-\mathrm{I}-\mathrm{Pb}$ angle linking adjacent octahedra deviates from $180^{\circ}$. For (PMA $)_{2} \mathrm{PbI}_{4}$, the in-plane $\mathrm{Pb}-\mathrm{I}-\mathrm{Pb}$ angle is approximately $158^{\circ}$, indicating a substantial distortion of the perovskite sheet. Increasing the length of the organic ligand by one carbon atom in $(\mathrm{PEA})_{2} \mathrm{PbI}_{4}$, reduces the in-plane $\mathrm{Pb}-\mathrm{I}-\mathrm{Pb}$ angle to approximately $153^{\circ}$. This means that the $\mathrm{PbI}_{6}$-octahedra are more rotated with respect to (PMA) $)_{2} \mathrm{PbI}_{4}$. Previous work ${ }^{25}$ has shown a blue-shift of the excitonic bands with decreasing dimensionality of the structure. A recent DFT study ${ }^{18}$ of $3 \mathrm{D}$ lead iodide structures showed a relation between the $\mathrm{Pb}-\mathrm{I}-\mathrm{Pb}$ bond angles and the steric size of the organic cations, as well as between the band gap and this bond angle. The band gap increases with increasing distortion (i.e., smaller $\mathrm{Pb}-\mathrm{I}-\mathrm{Pb}$ bond angles) by increasing the size of the organic molecules. Related work on $2 \mathrm{D}$ hybrid materials by Knutson et al. ${ }^{50}$ reveals the same trend. They studied 2D tin iodide hybrids and showed that the variation of the in-plane $S n-I-S n$ bond angle has the largest impact on tuning of the band gap. Once rotation occurs, the top of the valence band will be lowered as the antibonding interactions between the Sn-s and I- $p_{x}$ and $p_{y}$ orbitals are reduced. On the other hand, the bottom of the conduction band is raised as the distortion lowers the symmetry of the lattice. This results in significant antibonding between the Sn- $p$ and I-s/ $p$ hybrid orbitals in the conduction band. Thus, the increase in $\mathrm{Sn}-\mathrm{I}-\mathrm{Sn}$ bond angle distortion leads to an increase in the band gap. Our (PMA) ${ }_{2} \mathrm{PbI}_{4}$ and (PEA) ${ }_{2} \mathrm{PbI}_{4}$ compounds are in good agreement with this. We show experimentally that increasing the length of the organic cation increases the inplane distortion of the inorganic lead iodide sheets, which in turn induces a blue-shift in photoluminescence. Although this explains the blue-shift observed by going from PMA to PEA as the organic ligand, increasing the length to PPA and PBA is more complex. As the (PMA) $)_{2} \mathrm{PbI}_{4}$ and $(\mathrm{PEA})_{2} \mathrm{PbI}_{4}$ compounds are structurally similar (layers of corner-sharing $\mathrm{PbI}_{6}$ octahedra) and the (PPA $)_{3} \mathrm{~Pb}_{2} \mathrm{I}_{7}$ and $(\mathrm{PBA})_{3} \mathrm{~Pb}_{2} \mathrm{I}_{7}$ compounds are structurally similar as well (layers of both corner- and phasesharing $\mathrm{PbI}_{6}$-octahedra), we investigate the difference between $(\mathrm{PMA})_{2} \mathrm{PbI}_{4}$ and $(\mathrm{PPA})_{3} \mathrm{~Pb}_{2} \mathrm{I}_{7}$ using electronic structure calculations.

In Figure 4a, we show the band structure of (PMA) ${ }_{2} \mathrm{PbI}_{4}$. The band edges exhibit highly dispersive conduction and
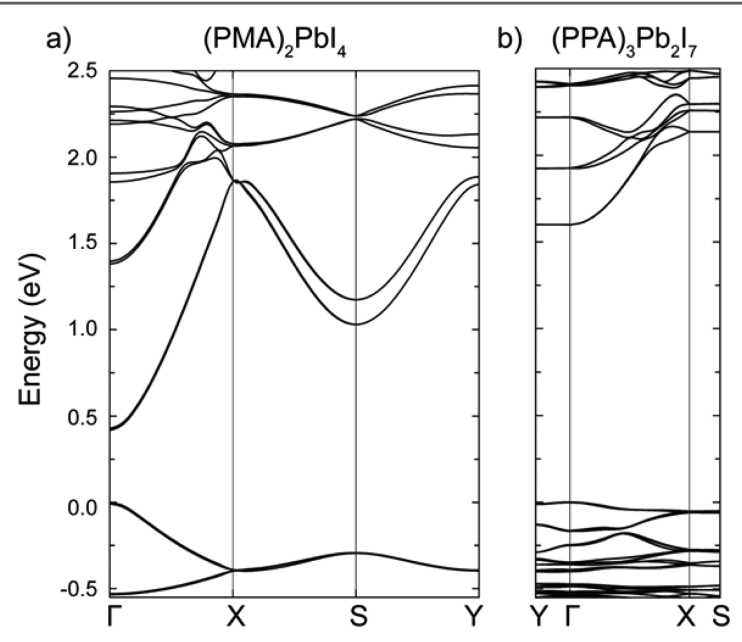

Figure 4. Electronic band structures of (PMA) ${ }_{2} \mathrm{PbI}_{4}$ (a) and (PPA) ${ }_{3} \mathrm{~Pb}_{2} \mathrm{I}_{7}$ (b) calculated within DFT+SOC. (a) The band structure of (PMA) ${ }_{2} \mathrm{PbI}_{4}$ is calculated along the high symmetry $\Gamma(0,0,0)-\mathrm{X}(\pi /$ $a, 0,0)-S(\pi / a, \pi / b, 0)-\mathrm{Y}(0, \pi / b, 0)$, where $a$ and $b$ are the lattice parameters along the $[100]$ and $[010]$ directions. At the $\Gamma$ point, the valence band top is predominantly of I-p (69\%) and Pb-s (26\%) character, whereas the conduction band bottom has a predominant $\mathrm{Pb}$ $p(69 \%)$ and I- $p(16 \%)$ character. Similarly, at the $S$ point the character of the valence band top is of Pb-s (21\%), I- $p$ (72\%) whereas the conduction band bottom consists of Pb-p (63\%) and I- $p$ (17\%). (b) The band structure of $(\mathrm{PPA})_{3} \mathrm{~Pb}_{2} \mathrm{I}_{7}$ is calculated along the high symmetry path $\mathrm{Y}(0, \pi / b, 0)-\Gamma(0,0,0)-\mathrm{X}(\pi / a, 0,0)-S(\pi / a, \pi / b, 0)$, where $a$ and $b$ are the lattice parameters along the [100] and [010] directions, respectively. All energies are referred to the top of the valence band in both panels a and $b$.

valence band edges within the (001) plane, and flat band edges along the [001] direction (not shown in Figure 4). This behavior is consistent with the quasi-2D character of the $(\mathrm{PMA})_{2} \mathrm{PbI}_{4}$ structure. The calculated band gap is direct at the $\Gamma$ point. We obtain a band gap of $0.42 \mathrm{eV}$ within DFT+SOC. The electron and hole effective masses in the (001) plane are small and isotropic. We obtain $0.24 \mathrm{~m}_{\mathrm{e}}$ and $0.15 \mathrm{~m}_{\mathrm{e}}$ for holes and electrons, respectively. The effective masses and the band 
$(\mathrm{PMA})_{2} \mathrm{Pbl}_{4}$

a) VBT

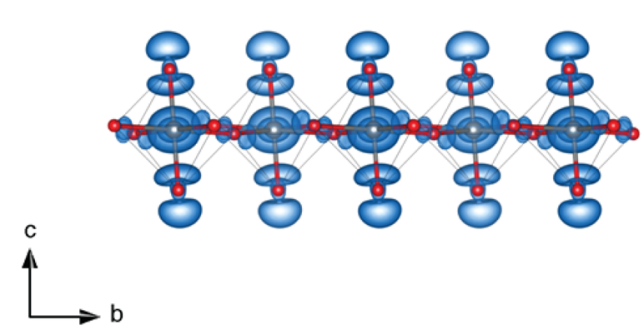

b) $\mathrm{CBB}$
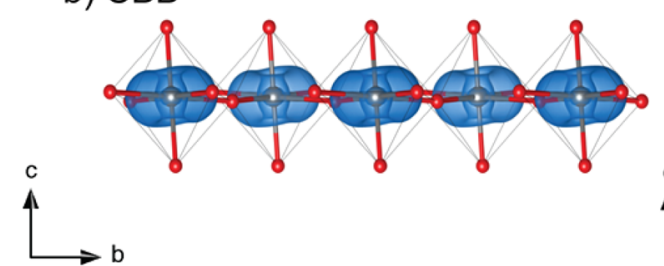

c) VBT
$(\mathrm{PPA})_{3} \mathrm{~Pb}_{2} \mathrm{I}_{7}$

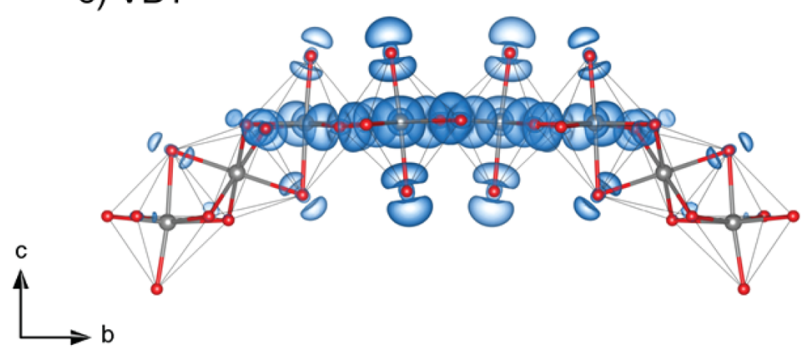

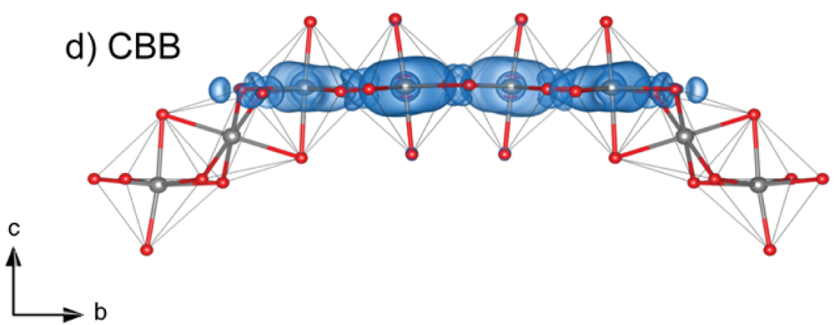

Figure 5. Spatial distribution of the electronic wave function for the top of the valence band (VBT) and bottom of the conduction band (CBB) at the $\Gamma$ point for (PMA) ${ }_{2} \mathrm{PbI}_{4}(\mathrm{a}, \mathrm{b})$ and $(\mathrm{PPA})_{3} \mathrm{~Pb}_{2} \mathrm{I}_{7}(\mathrm{c}, \mathrm{d})$. In both structures, the shape of the electronic wave functions is consistent with the I- $p$, $\mathrm{Pb}$ $p$ characters for the VBT and $\mathrm{CBB}$, respectively. In the case of $(\mathrm{PPA})_{3} \mathrm{~Pb}_{2} \mathrm{I}_{7}$, the charge is distributed only over the region containing corner-sharing octahedra, for both the VBT and CBB. For clarity, we show only the inorganic part of the structures, although the calculations were performed by including the organic ligands.

gap of (PMA $)_{2} \mathrm{PbI}_{4}$ are very similar to those obtained for bulk $\mathrm{CH}_{3} \mathrm{NH}_{3} \mathrm{PbI}_{3}$ within the same level of theory $(0.5 \mathrm{eV}$ band gap and effective masses of $0.13 \mathrm{~m}_{\mathrm{e}}$ and $0.11 \mathrm{~m}_{\mathrm{e}}$, for holes and electrons respectively). ${ }^{51}$ This finding is also fully consistent with previous calculations on monolayer and bilayer $\mathrm{CH}_{3} \mathrm{NH}_{3} \mathrm{PbI}_{3}{ }^{52}$ In Figure $4 \mathrm{~b}$, we show the band structure of $(\mathrm{PPA})_{3} \mathrm{~Pb}_{2} \mathrm{I}_{7}$. In contrast with (PMA) $)_{2} \mathrm{PbI}_{4}$, the band structure exhibits a dispersive profile only along the $\Gamma$ - X direction. This qualitative difference can be linked to the crystal structures of $(\mathrm{PPA})_{3} \mathrm{~Pb}_{2} \mathrm{I}_{7}$ and (PMA) $)_{2} \mathrm{PbI}_{4}$. Indeed, the (PMA) ${ }_{2} \mathrm{PbI}_{4}$ structure is highly isotropic in the (001) plane, with the $\mathrm{PbI}_{6}$ octahedra sharing corners throughout. In the case of (PPA $)_{3} \mathrm{~Pb}_{2} \mathrm{I}_{7}$, the $\mathrm{Pb}-\mathrm{I}$ network appears as an alternation of face- and corner-sharing octahedra, along the [010] direction.

For $(\mathrm{PPA})_{3} \mathrm{~Pb}_{2} \mathrm{I}_{7}$, we calculate a direct band gap of $1.6 \mathrm{eV}$ at the $\Gamma$ point, and effective masses along the [100] direction of $2.35 m_{\mathrm{e}}$ for holes and $0.44 m_{\mathrm{e}}$ for electrons, respectively. Within $\mathrm{DFT}+\mathrm{SOC}$, we also obtain that the band gap of the (PPA $)_{3} \mathrm{~Pb}_{2} \mathrm{I}_{7}$ structure is blue-shifted with respect to that of (PMA $)_{2} \mathrm{PbI}_{4}$. The band gap difference of $1.2 \mathrm{eV}$ can be rationalized by the additional quantum confinement along the [010] direction. This effect can be estimated from a quantum well model as $\Delta E_{\mathrm{g}}=\hbar^{2} \pi^{2} /\left(2 m^{*} m_{\mathrm{e}} L^{2}\right)$, where $m^{*}$ is the electronic effective mass for the (PMA) $)_{2} \mathrm{PbI}_{4}$ structure, $m_{\mathrm{e}}$ is the electron mass and $L$ is the size of the region formed by corner-sharing $\mathrm{PbI}_{6}$-octahedra in the inorganic layer of $(\mathrm{PPA})_{3} \mathrm{~Pb}_{2} \mathrm{I}_{7}$. We estimated that $L \approx 14.9 \AA$ from the experimental crystal structure, as shown in Figure 2c,d. From this simple model, we can estimate that $\Delta E_{\mathrm{g}}=1.1 \mathrm{eV}$, which is close to the difference in band gaps calculated for (PMA) ${ }_{2} \mathrm{PbI}_{4}$ and $(\mathrm{PPA})_{3} \mathrm{~Pb}_{2} \mathrm{I}_{7}$ from DFT+SOC. Interestingly, the alternating face-sharing $\mathrm{PbI}_{6}$-octahedra appear to have a similar confining effect for the electronic structure along the [010] direction as the organic cations in the [001] direction. To confirm this assumption, we calculated the wave functions corresponding to the highest occupied and lowest unoccupied states for both
$(\mathrm{PMA})_{2} \mathrm{PbI}_{4}$ and $(\mathrm{PPA})_{3} \mathrm{~Pb}_{2} \mathrm{I}_{7}$. In Figure $5 \mathrm{a}-\mathrm{d}$, we show the comparison between the spatial distribution of the wave functions at the top of the valence band and bottom of the conduction band for ( $\mathrm{PMA})_{2} \mathrm{PbI}_{4}$ and $(\mathrm{PPA})_{3} \mathrm{~Pb}_{2} \mathrm{I}_{7}$. In the former, the wave functions are delocalized throughout all octahedral sites; in the latter, the wave functions are concentrated around the corner-sharing octahedra; this effectively defines 1D nanoribbons of electronic charge in $(\mathrm{PPA})_{3} \mathrm{~Pb}_{2} \mathrm{I}_{7}$. This behavior is consistent for both the conduction band top and valence band bottom of the two structures.

The calculated band gap difference between the two structures $(1.2 \mathrm{eV})$ is larger than the energy difference of the peaks in the PL spectrum corresponding to these structures (approximately $0.2 \mathrm{eV}$ ), as shown in Figure 3 and Table 1. This difference is likely due to the level of approximation employed here. We expect that by incorporating quasiparticle corrections and excitonic effects this discrepancy will be resolved. Given the extended size of the (PMA) $)_{2} \mathrm{PbI}_{4}$ and $(\mathrm{PPA})_{3} \mathrm{~Pb}_{2} \mathrm{I}_{7}$ unit cells, the inclusion of quasiparticle and electron-hole interaction effects is computationally prohibitive. For reference, in the case of $\mathrm{CH}_{3} \mathrm{NH}_{3} \mathrm{PbI}_{3}$ quasiparticle effects increase the DFT+SOC band gap by more than $1 \mathrm{eV} .^{51,53}$ In addition, low-dimensional structures are known to exhibit enhanced quasiparticle corrections and excitonic effects with respect to their bulk counterparts, due to the reduction of the dielectric screening. ${ }^{54}$

In contrast with three-dimensional lead halide perovskites, in $(\mathrm{PMA})_{2} \mathrm{PbI}_{4}$ and $(\mathrm{PPA})_{3} \mathrm{~Pb}_{2} \mathrm{I}_{7}$ the large cations disrupt the three-dimensional corner-sharing connectivity. This reorganization of the structure strongly impacts the electronic properties of the lead iodide, inducing an increase in the band gap due to quantum confinement effects, as well as a change in the band edge features. In a tight-binding model interpretation of the band structures, the dispersive profiles of the bands are linked to the large overlap between electronic wave functions localized at neighboring sites. In both structures, dispersive bands are 
identified along directions where the $\mathrm{PbI}_{6}$-octahedra are cornersharing. Flat bands are found for directions that are either perpendicular to the plane formed by the $\mathrm{Pb}-\mathrm{I}$ network $\left[(\mathrm{PMA})_{2} \mathrm{PbI}_{4}\right.$ and $\left.(\mathrm{PPA})_{3} \mathrm{~Pb}_{2} \mathrm{I}_{7}\right]$ or along the direction of the chains of face-sharing $\mathrm{PbI}_{6}$-octahedra $\left[(\mathrm{PPA})_{3} \mathrm{~Pb}_{2} \mathrm{I}_{7}\right]$. Along these directions, the overlap of the wave functions is limited by the presence of either the organic cation or the face-sharing octahedra chains which are shown to confine the wave functions (Figure 5). Therefore, the corresponding electronic bands exhibit a flat profile. Indeed, the hole effective masses calculated within DFT+SOC increase by up to 1 order of magnitude, whereas the electron effective masses are almost tripled when the structure of the inorganic network incorporates face-sharing $\mathrm{PbI}_{6}$-octahedra.

Thus, compared to the $3 \mathrm{D} \mathrm{CH} \mathrm{CH}_{3} \mathrm{PbI}_{3}$, confinement along the $[001]$ direction to form $2 \mathrm{D}$ structures induces a blue-shift. The presence of face-sharing $\mathrm{PbI}_{6}$-octahedra induces an additional confinement effect in the [010] direction to create an effective $1 \mathrm{D}$ structure that enhances the blue-shift originally obtained from the first confinement effect. This additional confinement effect, as shown by the absence of dispersion in the [010] direction in the band structure of Figure 4, explains the more blue character of (PPA) ${ }_{3} \mathrm{~Pb}_{2} \mathrm{I}_{7}$ compared to $(\mathrm{PMA})_{2} \mathrm{PbI}_{4}$. Following the same argument, $(\mathrm{PBA})_{3} \mathrm{~Pb}_{2} \mathrm{I}_{7}$ has a shorter region formed by corner-sharing $\mathrm{PbI}_{6}$-octahedra compared to (PPA) ${ }_{3} \mathrm{~Pb}_{2} \mathrm{I}_{7}$, as shown in Figure $2 \mathrm{c}$,d. This could qualitatively mean that the confinement effect along the [010] direction is enhanced in $(\mathrm{PBA})_{3} \mathrm{~Pb}_{2} \mathrm{I}_{7}$ and that an even larger band gap would be observed compared to $(\mathrm{PPA})_{3} \mathrm{~Pb}_{2} \mathrm{I}_{7}$. This is indeed observed in Figure 3.

\section{CONCLUSIONS}

In conclusion, we have synthesized high-quality single crystals of lead iodide hybrids with compositions $\mathrm{A}_{2} \mathrm{PbI}_{4}$ and $\mathrm{A}_{3} \mathrm{~Pb}_{2} \mathrm{I}_{7}$, where $A$ are four different phenylalkylammonium cations. We obtained novel low-dimensional crystal structures that combine corner-sharing with face-sharing $\mathrm{PbI}_{6}$-octahedra and form a new class of organic/inorganic hybrid materials. The inorganic layers in this class are composed of corner-shared $\left[\mathrm{M}_{3} \mathrm{X}_{10}\right]^{4-}$ and $\left[\mathrm{MX}_{4}\right]^{2-}$ units. We have shown that systematically increasing the length of the phenylalkylammonium chains induces a blueshift in the photoluminescence. We have calculated the electronic structure of $(\mathrm{PMA})_{2} \mathrm{PbI}_{4}$ and $(\mathrm{PPA})_{2} \mathrm{~Pb}_{3} \mathrm{I}_{7}$ within $\mathrm{DFT}+\mathrm{SOC}$ and found that both compounds have semiconducting band structures, with direct band gaps. In the case of $(\mathrm{PPA})_{2} \mathrm{~Pb}_{3} \mathrm{I}_{7}$, the band structure exhibits signatures of quantum confinement effects. The calculated band edges are flat along the confinement directions $([010])$ and the band gap is larger than (PMA) ${ }_{2} \mathrm{PbI}_{4}$. Moreover, the electronic wave functions corresponding to the states at the band edges are confined to the regions of the inorganic network containing corner-sharing octahedra. The large cations lead to a reorganization of the structure to include face-sharing as well as corner-sharing $\mathrm{PbI}_{6}$-octahedra. This structural rearrangement leads to drastic changes in the band structures compared to compounds exhibiting only corner-sharing octahedra. Our work provides insight into how chemical substitutions and confinement effects allow direct tuning of the optical band gap in organic/inorganic hybrid materials. Besides the possible interest for multijunction solar cells, this knowledge is important for the development of tunable hybrid perovskite-based light emitting diodes.

\section{ASSOCIATED CONTENT}

\section{Supporting Information}

The Supporting Information is available free of charge on the ACS Publications website at DOI: 10.1021/acs.chemmater.6b00809.

Crystallographic information files of (PMA) ${ }_{2} \mathrm{PbI}_{4}$, (PPA) ${ }_{3} \mathrm{~Pb}_{2} \mathrm{I}_{7}$ and $(\mathrm{PBA})_{3} \mathrm{~Pb}_{2} \mathrm{I}_{7}$ (CIF).

Crystallographic data and refinement parameters, thermal ellipsoid figures of the asymmetric unit of $(\mathrm{PMA})_{2} \mathrm{PbI}_{4},(\mathrm{PPA})_{3} \mathrm{~Pb}_{2} \mathrm{I}_{7}$ and $(\mathrm{PBA})_{3} \mathrm{~Pb}_{2} \mathrm{I}_{7}$, unit cell parameters of $(\mathrm{PPA})_{3} \mathrm{~Pb}_{2} \mathrm{I}_{7}$ at different temperatures, powder XRD patterns of $(\mathrm{PPA})_{3} \mathrm{~Pb}_{2} \mathrm{I}_{7}$ and $(\mathrm{PBA})_{3} \mathrm{~Pb}_{2} \mathrm{I}_{7}$, DSC data of (PBA) $)_{3} \mathrm{~Pb}_{2} \mathrm{I}_{7}$, and time-resolved photoluminescence spectra (PDF).

\section{AUTHOR INFORMATION}

\section{Corresponding Author}

*T. T. M. Palstra. E-mail: t.t.m.palstra@rug.nl.

Notes

The authors declare no competing financial interest.

\section{ACKNOWLEDGMENTS}

M.E.K. was supported by The Netherlands Organisation for Science NWO (Graduate Programme 2013, No. 022.005.006). H.-H.F. and M.A.L. acknowledge funding from European Research Council (ERC Starting Grant "Hy-SPOD" No. 306983). M.R.F. and F.G. were supported by the Leverhulme Trust (Grant RL-2012-001), the U.K. Engineering and Physical Sciences Research Council (Grant No. EP/J009857/1, EP/ M020517/1), and the Graphene Flagship (EU FP7 Grant No. 604391). M.R.F. and F.G. acknowledge the use of the University of Oxford Advanced Research Computing (ARC) facility $^{39}$ and the ARCHER U.K. National Supercomputing Service under the "AMSEC" Leadership project in carrying out this work. We thank $H$. van der Velde for the elemental analysis.

\section{REFERENCES}

(1) Kojima, A.; Teshima, K.; Shirai, Y.; Miyasaka, T. Organometal Halide Perovskites as Visible-Light Sensitizers for Photovoltaic Cells. J. Am. Chem. Soc. 2009, 131, 6050-6051.

(2) Docampo, P.; Ball, J. M.; Darwich, M.; Eperon, G. E.; Snaith, H. J. Efficient Organometal Trihalide Perovskite Planar-Heterojunction Solar Cells on Flexible Polymer Substrates. Nat. Commun. 2013, 4, 2761.

(3) Chen, Q.; Zhou, H.; Hong, Z.; Luo, S.; Duan, H.-S.; Wang, H.H.; Liu, Y.; Li, G.; Yang, Y. Planar Heterojunction Perovskite Solar Cells via Vapor Assisted Solution Process. J. Am. Chem. Soc. 2014, 136, 622-625.

(4) Liu, D.; Kelly, T. L. Perovskite Solar Cells with a Planar Heterojunction Structure Prepared Using Room-Temperature Solution Processing Techniques. Nat. Photonics 2013, 8, 133-138.

(5) Liu, M.; Johnston, M. B.; Snaith, H. J. Efficient Planar Heterojunction Perovskite Solar Cells by Vapour Deposition. Nature 2013, 501, 395-398.

(6) Ke, W.; Fang, G.; Wan, J.; Tao, H.; Liu, Q.; Xiong, L.; Qin, P.; Wang, J.; Lei, H.; Yang, G.; et al. Efficient Hole-Blocking Layer-Free Planar Halide Perovskite Thin-Film Solar Cells. Nat. Commun. 2015, 6, 6700 .

(7) Zhang, W.; Saliba, M.; Moore, D. T.; Pathak, S. K.; Hörantner, M. T.; Stergiopoulos, T.; Stranks, S. D.; Eperon, G. E.; Alexander-Webber, J. A.; Abate, A.; et al. Ultrasmooth Organic-inorganic Perovskite Thin-Film Formation and Crystallization for Efficient Planar Heterojunction Solar Cells. Nat. Commun. 2015, 6, 6142. 
(8) National Renewable Energy Laboratory (NREL) Research Cell Efficiency Records, http://www.nrel.gov/ncpv/images/efficiency_ chart.jpg.

(9) Tan, Z.-K.; Moghaddam, R. S.; Lai, M. L.; Docampo, P.; Higler, R.; Deschler, F.; Price, M.; Sadhanala, A.; Pazos, L. M.; Credgington, D.; et al. SI: Bright Light-Emitting Diodes Based on Organometal Halide Perovskite. Nat. Nanotechnol. 2014, 9, 687-692.

(10) Zhang, F.; Zhong, H.; Chen, C.; Wu, X.; Hu, X.; Huang, H.; Han, J.; Zou, B.; Dong, Y. Brightly Luminescent and Color-Tunable Colloidal $\mathrm{CH}_{3} \mathrm{NH}_{3} \mathrm{X}_{3}(\mathrm{X}=\mathrm{Br}, \mathrm{I}, \mathrm{Cl})$ Quantum Dots: Potential Alternatives for Display Technology. ACS Nano 2015, 9, 4533-4542.

(11) Xing, G.; Mathews, N.; Lim, S. S.; Yantara, N.; Liu, X.; Sabba, D.; Grätzel, M.; Mhaisalkar, S.; Sum, T. C. Low-Temperature Solution-Processed Wavelength-Tunable Perovskites for Lasing. Nat. Mater. 2014, 13, 476-480.

(12) Zhu, H.; Fu, Y.; Meng, F.; Wu, X.; Gong, Z.; Ding, Q.; Gustafsson, M. V.; Trinh, M. T.; Jin, S.; Zhu, X.-Y. Lead Halide Perovskite Nanowire Lasers with Low Lasing Thresholds and High Quality Factors. Nat. Mater. 2015, 14, 636-642.

(13) Fang, Y.; Dong, Q.; Shao, Y.; Yuan, Y.; Huang, J. Highly Narrowband Perovskite Single-Crystal Photodetectors Enabled by Surface-Charge Recombination. Nat. Photonics 2015, 9, 679-686.

(14) Brivio, F.; Walker, A. B.; Walsh, A. Structural and Electronic Properties of Hybrid Perovskites for High-Efficiency Thin-Film Photovoltaics from First-Principles. APL Mater. 2013, 1, 042111.

(15) Filip, M. R.; Giustino, F. Computational Screening of Homovalent Lead Substitution in Organic-Inorganic Halide Perovskites. J. Phys. Chem. C 2016, 120, 166-173.

(16) Eperon, G. E.; Stranks, S. D.; Menelaou, C.; Johnston, M. B.; Herz, L. M.; Snaith, H. J. Formamidinium Lead Trihalide: A Broadly Tunable Perovskite for Efficient Planar Heterojunction Solar Cells. Energy Environ. Sci. 2014, 7, 982-988.

(17) Noh, J. H.; Im, S. H.; Heo, J. H.; Mandal, T. N.; Seok, S. Il. Chemical Management for Colorful, Efficient, and Stable InorganicOrganic Hybrid Nanostructured Solar Cells. Nano Lett. 2013, 13, 1764-1769.

(18) Filip, M. R.; Eperon, G. E.; Snaith, H. J.; Giustino, F. Steric Engineering of Metal-Halide Perovskites with Tunable Optical Band Gaps. Nat. Commun. 2014, 5, 5757.

(19) Smith, I. C.; Hoke, E. T.; Solis-ibarra, D.; Mcgehee, M. D.; Karunadasa, H. I. Perovskite Solar Cells A Layered Hybrid Perovskite Solar-Cell Absorber with Enhanced Moisture Stability. Angew. Chem., Int. Ed. 2014, 53, 11232-11235.

(20) Mitzi, D. B.; Wang, S.; Feild, C. A.; Chess, C. A.; Guloy, A. M. Conducting Layered Organic-Inorganic Halides Containing < 110>Oriented Perovskite Sheets. Science 1995, 267, 1473-1476.

(21) Takeoka, Y.; Fukasawa, M.; Matsui, T.; Kikuchi, K.; Rikukawa, M.; Sanui, K. Intercalated Formation of Two-Dimensional and MultiLayered Perovskites in Organic Thin Films. Chem. Commun. 2005, 378-380.

(22) Polyakov, A. O.; Arkenbout, A. H.; Baas, J.; Blake, G. R.; Meetsma, A.; Caretta, A.; Van Loosdrecht, P. H. M.; Palstra, T. T. M. Coexisting Ferromagnetic and Ferroelectric Order in a $\mathrm{CuCl}_{4}$-Based Organic-Inorganic Hybrid. Chem. Mater. 2012, 24, 133-139.

(23) Mitzi, D. B. Templating and Structural Engineering in Organicinorganic Perovskites. J. Chem. Soc. Dalt. Trans. 2001, 1-12.

(24) Ishihara, T. Optical Properties of PbI-Based Perovskite Structures. J. Lumin. 1994, 60-61, 269-274.

(25) Papavassiliou, G. C. Synthetic Three- and Lower-Dimensional Semiconductors Based on Inorganic Units. Mol. Cryst. Liq. Cryst. Sci. Technol., Sect. A 1996, 286, 231-238.

(26) Zhang, S.; Lanty, G.; Lauret, J. S.; Deleporte, E.; Audebert, P.; Galmiche, L. Synthesis and Optical Properties of Novel OrganicInorganic Hybrid Nanolayer Structure Semiconductors. Acta Mater. 2009, 57, 3301-3309.

(27) Calabrese, J.; Jones, N. L.; Harlow, R. L.; Herron, N.; Thorn, D. L.; Wang, Y. Preparation and Characterization of Layered Lead Halide Compounds. J. Am. Chem. Soc. 1991, 113, 2328-2330.
(28) Mitzi, D. B. Synthesis, Crystal Structure, and Optical and Thermal Properties of $\left(\mathrm{C}_{4} \mathrm{H}_{9} \mathrm{NH}_{3}\right)_{2} \mathrm{MI}_{4}(\mathrm{M}=\mathrm{Ge}, \mathrm{Sn}, \mathrm{Pb})$. Chem. Mater. 1996, 8 (3), 791-800.

(29) Cao, D. H.; Stoumpos, C. C.; Farha, O. K.; Hupp, J. T.; Kanatzidis, M. G. 2D Homologous Perovskites as Light-Absorbing Materials for Solar Cell Applications. J. Am. Chem. Soc. 2015, 137, $7843-7850$

(30) Mitzi, D. B. A Layered Solution Crystal Growth Technique and the Crystal Structure of $\left(\mathrm{C}_{6} \mathrm{H}_{5} \mathrm{C}_{2} \mathrm{H}_{4} \mathrm{NH}_{3}\right)_{2} \mathrm{PbCl}_{4}$. J. Solid State Chem. 1999, 145, 694-704.

(31) Altomare, A.; Camalli, M.; Cuocci, C.; Giacovazzo, C.; Moliterni, A.; Rizzi, R. EXPO2009: Structure Solution by Powder Data in Direct and Reciprocal Space. J. Appl. Crystallogr. 2009, 42, $1197-1202$

(32) Von Dreele, R; Larson, A. General Structure Analysis System (GSAS); Report No. LAUR 86-748; Los Alamos National Laboratory: Los Alamos, NM, 2004.

(33) Sheldrick, G. M. SHELXL97, Program for Crystal Structure Refinement; University of Göttingen: Göttingen, Germany, 1997.

(34) Papavassiliou, G. C.; Mousdis, G. A.; Koutselas, I. B. Some New Organic-Inorganic Hybrid Semiconductors Based on Metal Halide Unit: Structural, Optical and Related Properties. Adv. Mater. Opt. Electron. 1999, 9, 265-271.

(35) Wei, Y.; Audebert, P.; Galmiche, L.; Lauret, J. S.; Deleporte, E. Photostability of 2D Organic-Inorganic Hybrid Perovskites. Materials 2014, 7, 4789-4802.

(36) Kikuchi, K.; Takeoka, Y.; Rikukawa, M.; Sanui, K. Structure and Optical Properties of Lead Iodide Based Two-Dimensional Perovskite Compounds Containing Fluorophenethylamines. Curr. Appl. Phys. 2004, 4, 599-602.

(37) Billing, D. G.; Lemmerer, A. Catena-poly[tetrakis(3-Phenylpropyl-Ammonium) [iodoplumbate(II)-Tri-U-Iodo-plumbate(II)-TriU-Iodo-Iodo-plumbate(II)-Di-U-Iodo]]. Acta Crystallogr., Sect. C: Cryst. Struct. Commun. 2006, 62, m174-m176.

(38) Wiest, T.; Blachnik, R.; Reuter, H. Die Kristallstruktur von $\left[\mathrm{PhMe}_{3} \mathrm{~N}\right]_{4}\left[\mathrm{~Pb}_{3} \mathrm{Br}_{10}\right]$. Z. Naturforsch. 1999, 54 b, 1099-1102.

(39) Lode, C.; Krautscheid, H. Iodostannate Mit Polymeren Anionen: $\left(\mathrm{Me}_{3} \mathrm{PhN}\right)_{4}{ }_{4}^{2}\left[\mathrm{Sn}_{3} \mathrm{I}_{10}\right], \quad\left[\mathrm{Me}_{2} \mathrm{HN}-\left(\mathrm{CH}_{2}\right)_{2}-\mathrm{NMe}_{2} \mathrm{H}\right]_{2}{ }^{1}\left[\mathrm{Sn}_{3} \mathrm{I}_{10}\right]$ Und $\left[\mathrm{Me}_{2} \mathrm{HN}-(\mathrm{CH}\right.$. Z. Anorg. Allg. Chem. 2001, 627, 1454-1458.

(40) Stoumpos, C. C.; Malliakas, C. D.; Kanatzidis, M. G. Semiconducting Tin and Lead Iodide Perovskites with Organic Cations: Phase Transitions, High Mobilities, and near-Infrared Photoluminescent Properties. Inorg. Chem. 2013, 52, 9019-9038.

(41) Dong, Q.; Fang, Y.; Shao, Y.; Mulligan, P.; Qiu, J.; Cao, L.; Huang, J. Electron-Hole Diffusion Lengths > $175 \mathrm{Mm}$ in SolutionGrown $\mathrm{CH}_{3} \mathrm{NH}_{3} \mathrm{PbI}_{3}$ Single Crystals. Science 2015, 347, 967-970.

(42) Saidaminov, M. I.; Abdelhady, A. L.; Murali, B.; Alarousu, E.; Burlakov, V. M.; Peng, W.; Dursun, I.; Wang, L.; He, Y.; Maculan, G.; et al. High-Quality Bulk Hybrid Perovskite Single Crystals within Minutes by Inverse Temperature Crystallization. Nat. Commun. 2015, 6, 7586 .

(43) Fang, H.-H.; Raissa, R.; Abdu-aguye, M.; Adjokatse, S.; Blake, G. R.; Even, J.; Loi, M. A. Photophysics of Organic-Inorganic Hybrid Lead Iodide Perovskite Single Crystals. Adv. Funct. Mater. 2015, 25, 2378-2385.

(44) Fu, Y.; Meng, F.; Rowley, M. B.; Thompson, B. J.; Shearer, M. J.; Ma, D.; Hamers, R. J.; Wright, J. C.; Jin, S. Solution Growth of Single Crystal Methylammonium Lead Halide Perovskite Nanostructures for Optoelectronic and Photovoltaic Applications. J. Am. Chem. Soc. 2015, 137, 5810-5818.

(45) Papavassiliou, G. C.; Koutselas, I. R. Structural, Optical and Related Properties of Some Natural Three- and Lower-Dimensional Semiconductor Systems. Synth. Met. 1995, 71, 1713-1714.

(46) Kitazawa, N.; Watanabe, Y.; Nakamura, Y. Optical Properties of $\mathrm{CH}_{3} \mathrm{NH}_{3} \mathrm{PbX}_{3}(\mathrm{X}=$ Halogen $)$ and Their Mixed-Halide Crystals. $J$. Mater. Sci. 2002, 37, 3585-3587.

(47) Umeyama, D.; Lin, Y.; Karunadasa, H. I. Red-to-Black Piezochromism in a Compressible Pb-I-SCN Layered Perovskite. Chem. Mater. 2016, 28, 3241-3244. 
(48) Xiao, Z.; Meng, W.; Saparov, B.; Duan, H.; Wang, C.; Feng, C.; Liao, W.; Ke, W.; Zhao, D.; Wang, J.; et al. Photovoltaic Properties of Two-Dimensional $\left(\mathrm{CH}_{3} \mathrm{NH}_{3}\right)_{2} \mathrm{~Pb}(\mathrm{SCN})_{2} \mathrm{I}_{2}$ Perovskite: A Combined Experimental and Density Functional Theory Study. J. Phys. Chem. Lett. 2016, 7, 1213-1218.

(49) Papavassiliou, G. C.; Koutselas, I. B.; Terzis, A.; Whangbo, M.H. Structural and Electronic Properties of the Natural. Solid State Commun. 1994, 91 (9), 695-698.

(50) Knutson, J. L.; Martin, J. D.; Carolina, N.; Uni, S.; Mitzi, D. B.; Watson, I. B. M. T. J.; Box, P. O.; Heights, Y.; York, N. Tuning the Band Gap in Hybrid Tin Iodide Perovskite Semiconductors Using Structural Templating. Inorg. Chem. 2005, 44 (13), 4699-4705.

(51) Filip, M. R.; Verdi, C.; Giustino, F. GW Band Structures and Carrier Effective Masses of $\mathrm{CH}_{3} \mathrm{NH}_{3} \mathrm{PbI}_{3}$ and Hypothetical Perovskites of the Type $\mathrm{APbI}_{3}: \mathrm{A}=\mathrm{NH}_{4}, \mathrm{PH}_{4}, \mathrm{AsH}_{4}$, and $\mathrm{SbH}_{4}$. J. Phys. Chem. C 2015, 119, 25209-25219.

(52) Volonakis, G.; Giustino, F. Ferroelectric Graphene-Perovskite Interfaces. J. Phys. Chem. Lett. 2015, 6, 2496-2502.

(53) Filip, M. R.; Giustino, F. GW Quasiparticle Band Gap of the Hybrid Organic-Inorganic Perovskite $\mathrm{CH}_{3} \mathrm{NH}_{3} \mathrm{PbI}_{3}$ : Effect of SpinOrbit Interaction, Semicore Electrons, and Self-Consistency. Phys. Rev. B: Condens. Matter Mater. Phys. 2014, 90, 245145.

(54) Caruso, F.; Filip, M. R.; Giustino, F. Excitons in OneDimensional van Der Waals Materials: $\mathrm{Sb}_{2} \mathrm{~S}_{3}$ Nanoribbons. Phys. Rev. B: Condens. Matter Mater. Phys. 2015, 92, 125134. 